\title{
Philosophiques
}

\section{La république face à la question de la race}

\section{Luc Foisneau}

Volume 40, numéro 2, automne 2013

URI : https://id.erudit.org/iderudit/1023709ar

DOI : https://doi.org/10.7202/1023709ar

Aller au sommaire du numéro

Éditeur(s)

Société de philosophie du Québec

ISSN

0316-2923 (imprimé)

1492-1391 (numérique)

Découvrir la revue

Citer ce document

Foisneau, L. (2013). La république face à la question de la race. Philosophiques, 40(2), 479-485. https://doi.org/10.7202/1023709ar d'utilisation que vous pouvez consulter en ligne.

https://apropos.erudit.org/fr/usagers/politique-dutilisation/ 


\title{
La république face à la question de la race
}

\author{
LUC FOISNEAU \\ Centre d'Études Sociologiques et Politiques Raymond Aron - CNRS \\ EHESS \\ luc.foisneau@ehess.fr
}

Le livre de Magali Bessone arrive à point nommé dans le débat intellectuel français. Alors qu'en Amérique du Nord, aux États-Unis depuis le mouvement des droits civiques, mais aussi au Canada, la discussion sur la question de la «race» fait rage depuis de nombreuses années, la France a pris en la matière un retard d'autant plus regrettable que le non-dit oriente une bonne partie du débat politique français sur l'immigration, les "minorités visibles ", les banlieues, l' "exclusion" et, très récemment encore, sur les Roms. Ce non-dit devient d'autant plus assourdissant que, les effets sociaux de la crise économique se faisant davantage sentir, il fait retour dans la politique sous la forme de ce que l'on nomme improprement le "populisme". Tout se passe comme si seuls les partis "populistes », ou ceux qui dans les autres partis leur emboîtent le pas, étaient désormais capables de parler d'un réel refoulé, la réalité sociale racialisée.

Sans distinction de race? a ainsi le grand mérite d'annoncer la couleur: on ne peut plus faire comme si les "races» n'existaient pas, nous est-il dit, puisque, en dépit de leur inexistence avérée d'un point de vue biologique, elles contribuent à façonner notre réalité sociale. On ne peut continuer à faire comme si, parmi d'autres caractéristiques de notre phénotype, la couleur de notre peau n'avait aucune espèce d'incidence sur nos vies. Tout le monde sait d'un savoir commun confirmé par des enquêtes, encore peu nombreuses en France où les statistiques ethniques sont interdites, que le fait d'être "black, blanc ou beur » conduit en général à des parcours sociaux différenciés, car les discriminations se manifestent aussi bien à l'école que sur le marché du travail, et celui du logement. Le livre de Magali Bessone part de ce savoir commun pour revendiquer haut et fort la nécessité d'une recherche en sciences sociales qui prenne pour objet d'étude les phénomènes sociaux liés à la «race». Dans le contexte français, où les sciences sociales commencent à rattraper sur ce point leur retard ${ }^{1}$, son originalité est toutefois de revendiquer cette nécessité pour la philosophie et, en particulier, pour la philosophie normative.

L'objection qui vient immédiatement à l'esprit d'un philosophe politique français nourri au républicanisme est la suivante: comment peut-on

1. P. N'Diaye, La condition noire, Paris, Calmann-Lévy, 2008; Didier Fassin, Éric Fassin, De la question sociale à la question raciale? Représenter la société française, Paris, La Découverte, 2006. 
justifier que l'on puisse adopter, fut-ce pour le surmonter, le point de vue de la «race»? Que les sciences sociales aient à en passer par là pour dissiper un non-dit qui pèse lourd sur la vie de milliers de personnes est compréhensible: donner de la notion de «race» une définition rigoureuse et publier des enquêtes sur ce sujet, c'est rendre lisibles des phénomènes bien réels qui autrement passeraient inaperçus. Mais pourquoi, dira notre philosophe, faudrait-il prendre en considération cette notion dans le champ de la philosophie moraleet politique? Si les valeurs de la république sont aux antipodes du racisme, n'est-il pas préférable de refuser à la race droit de cité dans notre constitutionet dans notre espace public?

La question que je voudrais poser à Magali Bessone concerne la manière dont elle articule les deux niveaux de son analyse, celui des normes et celui des sciences sociales. Je souhaiterais, notamment, mesurer la distance qui sépare sa position de ce que l'on pourrait appeler le républicanisme libéral. Plus encore que le multiculturalisme dans ses variantes radicales et libérales (p. 195-206), le républicanisme classique constitue en effet, dans le cadre de sa démonstration, une cible de choix. S'il y a bien sûr d'autres bases théoriques à la volonté de promouvoir une théorie critique de la race, l'une des motivations de Magali Bessone est une insatisfaction très grande à l'égard des arguments du républicanisme en matière de discriminations raciales. Bien que cette insatisfaction soit partagée, il me semble qu'en comprendre la nature exacte, dans l'argument de ce livre, pourrait nous permettre de mieux apprécier la portée de Sans distinction de race?

\section{Les raisons de la colère (à l'encontre du républicanisme libéral)}

Précisons tout d'abord l'objet de cette insatisfaction: derrière de belles Déclarations (des droits de l'homme et du citoyen), le républicanisme classique serait incapable de porter remède aux discriminations raciales, car l'universalisme et l'individualisme qui le fondent rendraient impensable l'erreur catégorielle consistant à discriminer —-distinguer, mais aussi, au figuré, juger et mettre à part- une personne en raison de sa "race». Si une telle partialité existe aussi bien en matière de "genre» et de "classe sociale », elle est plus particulièrement pernicieuse dans le cas de la «race», car elle s'exerce alors, en régime républicain, sous un voile d'ignorance particulièrement épais : comment pourrait-on espérer remédier à un travers que la loi va jusqu'à nous interdire de nommer? On peut, certes, être poursuivi au titre de la loi Taubira pour "racisme", mais cette poursuite ne s'appuie pas sur une définition de la «race», puisque ce qui est punissable est de parler de quelqu'un ou à quelqu'un en faisant référence à sa « race». S'il ne suffit pas, toutefois, de bannir la notion de «race» du texte de la constitution française pour faire cesser les contrôles au faciès, c'est que les « races » existent à n'en pas douter dans les pratiques routinières de la police, et dans bien d'autres pratiques sociales. Si le républicanisme libéral suscite de plus en plus d'insatisfaction, c'est qu'il condamne le racisme, après nous avoir privé des moyens 
de comprendre la production sociale des races. Tour de passe-passe républicain libéral: faisons disparaître la race (de notre lexique constitutionnel et politique et, si possible, de nos opinions et de nos croyances), et le racisme disparaîtra aussitôt. Comme si l'on pouvait venir à bout d'un phénomène social complexe à partir de bonnes intentions (d'antiracisme) fondées sur des déclarations (des droits de l'homme)! Magali Bessone considère, à juste titre, que la meilleure manière de remédier à cet illusionnisme est, en s'appuyant sur les travaux des sciences sociales, de donner une place dans le discours public à la notion de "race». Une théorie rigoureuse de la "race» nous permettrait, selon elle, d'identifier les dysfonctionnements sociaux induits par le racisme. Prenons-en acte. Toutefois, la critique qu'elle adresse au républicanisme libéral remet-elle en cause l'horizon normatif au sein duquel s'inscrit cette dernière philosophie politique? Il est, en effet, nécessaire, me semble-t-il, de distinguer entre une insatisfaction légitime à l'égard de l'impuissance du républicanisme classique à venir à bout du racisme, que l'on ne peut que partager, et ce qui peut apparaître comme un rejet des valeurs qui animent cette philosophie (universalisme et individualisme). Dans quelle mesure la critique légitime de l'insuffisance républicaine à comprendre les phénomènes de «racisme» implique-t-elle une critique de son contenu normatif?

Si cette question me paraît mériter d'être posée, c'est que le propos de Magali Bessone l'esquive, présupposant en quelque sorte que la réponse irait de soi. Je me demande néanmoins si cette évidence ne participe pas elle aussi d'une certaine forme de rhétorique, celle de la Théorie critique, inverse de celle des droits de l'homme: à trop vouloir se distinguer du républicanisme libéral, cette théorie ne finirait-elle pas par oublier qu'elle partage avec lui un certain nombre de valeurs? Autrement dit, l'insuffisance des moyens théoriques et pratiques mis en œuvre par les républicains libéraux exigerait non seulement de prendre au sérieux la construction sociale de la notion de race pour mieux la déconstruire, mais aussi de renoncer une bonne fois pour toutes à l'universalisme et à l'individualisme qui sous-tendent la doctrine morale républicaine. Si j'accepte volontiers la première proposition, défendue avec beaucoup de brio par Magali Bessone, il me semble que la deuxième proposition mériterait à tout le moins une justification car, entre les deux propositions, il n'y a pas, me semble-t-il, le lien évident qu'elle présuppose.

\section{Un républicain peut-il être raciste dans le savoir?}

Le raisonnement implicite me semble être le suivant: si le citoyen républicain et l'individu libéral - je mets ici sur le même plan ces deux cousins germains de la pensée politique moderne- ne parviennent pas à comprendre les phénomènes de discrimination raciale, c'est que les valeurs dont ils sont porteurs en sont responsables. 
Magali Bessone considère, d'une part, que les principes universels et individualistes produisent une sorte d'aveuglement qui équivaut plus ou moins à autoriser - en leur donnant une sorte d'impunité - les conduites de discrimination, et, d'autre part, que les faits et les normes ne se situent pas sur un même plan - ce en quoi on ne peut que lui donner raison. De deux choses l'une: soit l'usage de normes inadéquates peut conduire à créer ou renforcer insidieusement des phénomènes de racialisation, soit ces phénomènes reposent sur des causes sociales distinctes des normes revendiquées par la république.

L'argument de Magali Bessone me semble porteur, en la matière, d'une certaine ambiguité. Si l'on a bien compris que l'objet du livre est "d'abord épistémique ", à savoir de montrer que «les races sont construites» et qu'en tant que telles «elles existent» (p. 223), l'analyse de la racialisation d'une société passe, dans l'argumentaire du chapitre VI, par la prise en considération des valeurs dont elle se revendique. Dès lors il n'est pas vrai que l'on puisse comprendre la place des « races » dans une république libérale comme la France indépendamment des valeurs inscrites dans sa constitution. On ne saurait donc séparer, comme Magali Bessone semble vouloir le faire lorsqu'elle expose les fondements moraux du républicanisme critique, qui a ses faveurs, le versant épistémique du versant normatif de son travail, puisque les normes de la république libérale contribuent très directement, si l'on suit son raisonnement, à la production de discriminations raciales.

Considérons la manière dont les deux niveaux de l'analyse, sociologique et normatif, se mêlent dans la théorie critique de Magali Bessone. Deux mécanismes sont à l'œuvre, nous dit-elle en substance, dans le républicanisme classique.

I. Le premier consiste à promouvoir une politique de non-discrimination, non pour corriger les effets de la discrimination comme on pourrait le croire naïvement, mais pour dissimuler dans la sphère publique le rôle joué dans nos sociétés démocratiques par les «races»:

Le discours officiel préfère parler, positivement, de promotion de la «diversité» ou d' "égalité des chances", sans que ces deux principes soient clairement définis, plutôt que d'affronter la lutte contre les inégalités, injustices et dominations raciales qui impliqueraient une refonte profonde du modèle républicain (p. 2I 8).

Dans cet anti-racisme officiel opère, selon l'heureuse formule de Magali Bessone, une «euphémisation des principes» (ibid.). Autrement dit, si l'on parle tant de racisme, c'est pour éviter de mettre en œuvre des politiques concrètes de lutte contre les discriminations. Stratégie subtile ou, plutôt, perverse, qui consiste à jeter un voile d'ignorance sur la réalité pour mieux laisser perdurer une logique de relégation fondée sur une hiérarchisation implicite entre les "races». Les normes supérieures que la république libérale invoque ne servent pas à guider l'action publique, mais à nous 
tromper: il s'agit de nous faire prendre les vessies des discours anti-racistes, d'autant moins généreux qu'ils sont plus généraux, pour les lanternes de politiques concrètes visant à interdire, par exemple, les discriminations à l'embauche. En l'occurrence, l'ignorance feinte de l'origine historique de telle ou telle discrimination produit un "effet de dissimulation» (p. 219). Et c'est l'invocation d'un principe normatif égalitariste, celui de nondiscrimination, qui produit, en l'occurrence, cette occultation. C'est donc bien, si l'on suit le raisonnement de Magali Bessone, que les normes et les valeurs ne sont pas étrangères au maintien des discriminations raciales; il convient donc d'en tenir compte dans l'analyse des phénomènes de racialisation. Dans sa composante libérale, l'affirmation des droits des individus, le républicanisme contribue donc à entretenir une situation de discrimination.

2. Un second mécanisme produit également des effets insidieux: dans sa composante communautaire, lorsqu'il entend défendre les droits d'un peuple particulier, le républicanisme contribue, selon Magali Bessone, à créer les conditions d'une discrimination politique. Reprenant à Olivier Le Cour Grandmaison le terme de "xénophobie d'État» (p. 2 I9), Magali Bessone rappelle que les républiques libérales mettent parfois en œuvre des politiques très peu libérales: c'est le cas lorsque l'on prend pour cible les Roms au motif qu'ils refuseraient de se conformer au modèle commun fixé par la citoyenneté républicaine idéale. On fait alors comme si la question de la «race» ne jouait aucun rôle, alors qu'elle est omniprésente derrière les discours sur l'immigration et sur l'identité nationale. Au prisme de l'«identité nationale", l'idée de citoyenneté produit un effet de discrimination dont l'origine, là encore, est bien normative.

On peut regretter que Magali Bessone n'approfondisse pas davantage le lien entre la racialisation de certaines catégories de Français rétives aux modes de vie de la majorité sociologique, et le discours normatif républicain. On peut regretter notamment qu'elle ne précise pas en quel sens, selon elle, "[1]a conception individualiste et universaliste des droits accompagne et soutient la croyance en l'unicité du peuple et en l'indivisibilité de la République» (p. 220). Que la croyance en l'unité d'un peuple puisse s'accompagner, en régime républicain, du rejet des «minorités » - le traitement par la République française des minorités linguistiques et régionales en est un bon exemple -, mais dans quelle mesure l'universalité des droits de l'homme est-elle mise en cause par le mode particulier de constitution d'une telle communauté nationale? L'histoire de la domination de certains groupes racialisés - les gitans, les "arabes" et les "noirs" — dans une république comme la France peut-elle être imputée à la pensée des droits de l'homme? L'imbrication des normes morales et politiques présupposée par cette critique radicale ne remet-elle pas en cause la validité même du modèle républicain, fût-il accommodé à la mode américaine? 


\section{Les vertus du néo-républicanisme}

Telle n'est pas la thèse de Magali Bessone, qui entend préserver de la critique la fine pointe de la pensée normative républicaine. L'imbrication qui mine, selon elle, le républicanisme libéral n’aurait pas de prise sur le néorépublicanisme. À l'appui de son propos, elle s'efforce de montrer que la catégorie de respect ne saurait entretenir d'accointance douteuse avec les logiques de racialisation:

La norme du respect est fondamentale à la compréhension du concept de racisme et à son évaluation morale négative unifiée: quelle que soit la multiplicité des phénomènes racistes, il s'agit toujours de repérer des instances de manque de respect qui permettent d'évaluer moralement le défaut normatif dans les relations mises en place (p. I 84).

C'est là encore un concept normatif, paradoxalement, qui sert à définir un concept, celui de racisme, lequel devrait permettre une lecture sans fard de la réalité sociale. Mais suffit-il de passer du républicanisme classique au républicanisme critique, en l'occurrence celui de Cécile Laborde, pour qu'aussitôt se dissipe le risque d'un détournement des normes républicaines au profit de l'occultation publique de certains groupes, voire, de leur désignation à la vindicte populaire? On peut comprendre qu'une histoire des relations de domination au sein d'une république puisse servir à déciller les yeux des citoyens sur les tensions présentes dans leur communauté, mais suffit-il de parler le langage de la domination pour que l'on parvienne aussitôt à parler du respect et de la liberté de manière non idéologique? Si l'on n'a de cesse de mettre en avant les logiques de domination qui ont opposé certains groupes de citoyens par le passé, est-on certain de contribuer par là même à développer le respect entre les individus qui les composent? Si «les groupes en question existent dans et par le rapport social, la relation de domination ou de résistance à la domination » (p. 222), il n'est pas certain que l'on puisse rester très longtemps dans le cadre libéral de la république que présuppose aussi le néo-républicanisme, celui de Pettit notamment, avec son insistance sur la liberté comme non-domination. La conclusion d'une telle analyse critique ne serait-elle pas, plutôt que de promouvoir le respect de chacun pour chacun, d'inciter chacun à assurer à son groupe d'affiliation (quelle qu'en soit la définition, raciale ou religieuse) les moyens de sa prédominance au sein de la république? Je ne vois pas bien en quoi le fait de revendiquer la non-domination à titre individuel pourrait changer quoi que ce soit à cette conclusion très peu républicaine et, en aucun cas, libérale. Si la vérité de notre existence sociale réside dans notre appartenance à une ethnie ou à une religion, fût-ce dans le regard d'autrui, il peut paraître vain de vouloir nous garantir, à titre individuel, les conditions de notre liberté, puisque cette liberté fragile n'existe jamais que dans l'horizon de la domination d'un groupe par un autre. 
Une fois que l'on aura ouvert les yeux des républicains sur le caractère racialement construit de leur communauté politique et sur le caractère idéologique de leurs croyances normatives - c'est l'un des sens de la théorie critique de Magali Bessone -, il me paraît difficile d'échapper à la conclusion selon laquelle la défense des droits des individus passe, non par la défense de la république, mais par la défense des droits de groupes ethniques ou religieux. De ce point de vue, il est difficile de comprendre ce que pourrait être une théorie de la justice raciale ou, comme il est dit dans la conclusion, un «avenir "racialement" juste» (p. 223) : faut-il entendre par là une théorie de l'égalité qui passerait par un égal droit au respect pour chacune des «races " et des religions? Un droit à être fier d'être noir, gitan ou normand?

Une fois que l'on a réintroduit les races dans le discours public, y compris avec les garanties de sérieux que peut fournir la théorie sociale, il n'est pas certain que l'on parvienne à refonder, sur une telle base, une république du respect, encore moins une république de la sollicitude. La partie la plus difficile du programme de recherche de Magali Bessone me semble donc être devant elle: parvenir à reconstruire des normes communes sur les ruines laissées par la théorie critique de la race. 